\title{
Part C Early Intervention Providers' Knowledge of Autism Spectrum Disorder Prior To and Following ASD-Specific Training
}

\author{
Trisha L. Self, Darby Batchelor, Douglas F. Parham \\ Wichita State University, USA
}

\begin{abstract}
The American Academy of Pediatrics, recommendation that all children be screened for autism at 18 and 24 months has not been widely adopted by the medical community. Early intervention (EI) providers who work with high-risk children through Part $C$ of the Individuals with Disabilities Education Act (IDEA) can serve as an initial point of contact in detecting those at risk for autism spectrum disorder (ASD). Yet, EI providers have varied training backgrounds that affect their views regarding autism. The Autism Stigma and Knowledge Questionnaire (ASK-Q) was administered to EI providers prior to and following an ASD-specific training. Participants' responses to the $A S K-Q$ were analyzed to determine if participants' knowledge of ASD improved following the workshop. Results indicated that the professionals' overall knowledge of autism improved after attending the training. Further, it was determined participants' knowledge improved across the three knowledge subdomains of the ASK-Q. This supports the literature recommending that EI providers be trained in conducting ASD-specific screenings to assist with the early identification of autism.
\end{abstract}

\section{Introduction}

Autism Spectrum Disorder (ASD) is an earlyemergent neurodevelopmental disorder that is characterized by deficits in social communication and interaction skills, along with the presence of restrictive, repetitive patterns of behavior and interests [1]. Today, approximately 1 in 54 children below age 8 have been diagnosed with ASD [1].

Evidence has shown that ASD can be accurately diagnosed between 2-3 years of age; therefore, the early identification of children who demonstrate characteristics of ASD must become a priority for infant and toddler interventionists and healthcare providers. Conducting early screenings, using autism-specific screening tools, is critical to this identification process [2], [3].

Screenings can put families on the proper path to a timely and accurate diagnosis for their child. Further, early screenings can reduce the time caregivers remain uncertain about the diagnosis their child may receive. When children are identified early, they can gain access to targeted services, which can result in improved developmental outcomes [3].

\section{Review of the Literature}

Despite this documented evidence, and reports indicating that caregivers express concerns for their child's development between 15-19 months of age, the practice of screening young children for ASD has continued to lag [4]. In response, national initiatives and professional guidelines have been introduced, such as the Centers for Disease Control and Prevention "Learn the Signs. Act Early" campaign [1] and the American Academy of Pediatrics (AAP) practice guidelines for universal autism screenings for young children [5], [4].

The AAP guidelines specifically recommended that pediatricians screen for ASD using ASD-specific screening tools at 18 and 24 months and/or whenever a caregiver expresses a concern for ASD [5], [6]. It was further recommended that toddlers who receive a positive screen be referred to an early intervention program [5].

Survey data reveals, however, many pediatricians and family physicians do not screen children for ASD in accordance with the AAP guidelines [7]. Barriers identified by physicians to providing these services include, but are not limited to, a preference for relying on clinical observations, unfamiliarity with ASDspecific screening tools, limited time, inadequate reimbursement, and less than adequate ASD preprofessional education [7]. Consequently, children are not being diagnosed until 4-6 years of age, which means they might not be getting the help they need. This adversely affects quality of life for the child and family [8], [9].

The delay in early ASD screenings highlights the need to consider alternative approaches that capitalize on existing frontline service delivery systems for children under 3 years of age. The Part $\mathrm{C}$ early intervention (EI) system presents a possible infrastructure for implementing early screenings, providing timely and informed diagnostic referrals, and offering targeted services to young children who demonstrate characteristics of ASD [4].

Part C programs are available in most U.S. communities and services are provided to families 
regardless of their ability to pay. The Part $\mathrm{C}$ model was designed to be family-centered; that is, services and resources are determined for both the young children who qualify and their family. In so doing, attention is given to strengthening the family system, thereby increasing the likelihood of an improved developmental outcome for infants and toddlers. Because EI providers have on-going interactions with the children and their caregivers through the Part $\mathrm{C}$ programming, early and consistent relationships are typically established. Therefore, while EI providers cannot diagnose autism, they are well-positioned to offer families information and support to act quickly and persistently on behalf of young children with possible ASD and their caregivers [4].

Studies have confirmed, however, that professionals who work in infant/toddler programs (e.g., speechlanguage pathologists, occupational therapists, physical therapists, early education teachers) may not have a strong understanding of ASD-specific screening and evaluation procedures. This is due, in part, to a lack of education received at a pre-professional level on topics related to screening, assessing, and referring young children who present with characteristics of autism [10].

A report from Tomlin and colleagues revealed challenges EI providers had with conducting ASDscreenings. When surveyed, respondents indicated that they were more likely to administer a developmental screening than an ASD-specific screening. This was partially a reflection of the type of instruction they had received as a pre-professional [11]. Respondents also reported that they did not feel confident screening for autism when caregivers had not expressed autism as a concern, nor did they feel they had been sufficiently trained to discuss issues related to ASD with family members [11]. Pizur-Barnekow and colleagues surveyed Part $\mathrm{C}$ coordinators and identified barriers to screening for ASD among EI providers which included a lack of knowledge of ASD and ASD-specific screening tools, and a sense of uncertainty when discussing positive screening results with families [12]. As indicated by Harrison and colleagues, providers' lack of knowledge on issues related to recognizing the signs and symptoms of ASD as well as conducting ASD-specific screenings, often perpetuates disparities in the timing and quality of services received. In-depth quantification of ASD knowledge among EI providers may aid in the development of training programs that target specific, highly relevant topics [13].

Trainings that have focused on increasing professionals' knowledge of ASD by focusing on the value, use, and administration of ASD-specific screening protocols, have resulted in an increased use of those tools [14], [15]. It has also been reported that when workshop participants were trained to use the ASD-specific screening tools, versus those who were not, an increased use was reported. Further, the signs and symptoms of ASD were being identified at a younger age [14], [15].

Based on the documented evidence, instruction to assist Part $\mathrm{C}$ providers to conduct ASD-specific screenings should focus on improving their ability to recognize the signs and symptoms of ASD, conduct ASD-specific screenings, confidently discuss the results of an ASD-screening/evaluation with parents, and assist families to locate and secure appropriate resources as early as possible [9]. Poor knowledge and lack of proper education and training across various EI professions can impede early screening, accurate identification, and early referral of children with possible ASD [4].

The purpose of this study was to determine EI providers' knowledge of ASD prior to and following an ASD-specific instruction tailored to meet the needs of EI providers. The barriers and challenges to screening as reported in the literature by early interventionists, were considered when selecting topics and activities for this training [10], [11], [12]. Based on this informed input, the full-day workshop was structured to provide attendees with information on the characteristics of ASD, the importance and challenges of early screening, training on and simulated practice to implement an ASD-specific screening tool, the Modified Checklist for Autism in Toddlers-Revised with Follow Up (MCHAT-R/F) [16], and strategies for discussing screening results with family members.

\section{Method}

This study was approved by the Wichita State University Institutional Review Board. Participants were early interventionists who were recruited from a Part $\mathrm{C}$ early education program located in an urban Midwestern city.

\subsection{Survey Instrument}

The Autism Stigma and Knowledge Questionnaire (ASK-Q) [13] was used to determine participants' preand post-training knowledge of ASD. The ASK-Q consists of 49 survey items designed to comprehensively assess ASD knowledge while maintaining strong psychometric support and crosscultural utility. The ASK-Q items, derived from published research, are comprised of four subdomains. Three of the subdomains measure knowledge of ASD in the following areas: (1) diagnosis/symptoms (D/S, 17 items), (2) etiology (ET, 15), and (3) treatment (TR, 13), while the fourth determines stigma endorsement (ST, 7). Participants are asked to respond to survey statements by signifying if they "agree," "disagree," or "don't know." Cutoff scores on each subscale have been established. The cutoff scores were established as follows: in the D/S subdomain, a score of $0-10$ is considered inadequate knowledge of ASD, 11-17 is 
considered adequate; for ET, 0-10 is inadequate, 11- 15 is adequate; for TR, $0-9$ is inadequate, $10-13$ is adequate; in the ST subdomain, $0-2$ is within the "endorse stigma" subscore range, while a score of 3-7 is considered in the "do not endorse stigma" subscore range [13]. Demographic questions were also included on the survey. Participants were asked to provide the following information: gender, age range, professional discipline/role, practice setting, and number of years in practice following receipt of a post-secondary degree. Respondents were also asked if they routinely conducted ASD screenings, what screening tools they used, and the course of action taken following a positive screen. Finally, participants were asked to identify perceived barriers to screening for ASD (see Table 1 for participant demographic information).

\subsection{Participants}

Thirty-five early intervention providers, employed by an early intervention agency in Kansas, attended a full-day training titled, "ASD Screening Workshop for Early Interventionists." The Part C EI providers represented disciplines including early childhood education, speech-language pathology, physical therapy, occupational therapy, behavioral intervention, social work, and audiology. Prior to the workshop, all attendees were given the option to participate in this study, which consisted of completing the paper-based ASK-Q prior to and following completion of the training. Participants were informed that their decision to participate would not affect their relationship with the university. A total of 31 professionals consented to participate and completed both questionnaires. No identifying information was collected, and surveys were coded for pre- and post-workshop comparison.

\subsection{Procedure}

A faculty member from a local university with expertise in ASD conducted the workshop. The faculty member introduced the research project and the purpose for collecting the information. An administrator from the EI agency distributed the ASK$\mathrm{Q}$ to all attendees prior to and at the conclusion of the workshop. Those who chose to participate completed the questionnaires. The administrator collected the questionnaires and gave them to the faculty member at the conclusion of the training.

\subsection{Analysis}

A comparison of the before and after questionnaire responses for each participant was completed. For each statement, the participants had the option of responding "agree," "disagree," and "don't know." "Don't know" statements were coded as incorrect responses [13].
One coder entered the data from the 62 surveys into an Excel spreadsheet, then subsequently and separately re-entered the data from 20 of the 62 surveys to assess intra-rater reliability $(100 \%)$. A second coder independently cross-checked the data from 20 of the 62 surveys; these data were then independently compared to the first coder's spreadsheet to assess inter-rater reliability $(100 \%)$.

The survey statements prior to and following the training were analyzed to determine if the participants' overall knowledge of ASD had improved following the workshop. Subdomain scores were also calculated to determine if participants' knowledge in D/S, ET, TR or ST endorsement had changed.

\section{Results}

The first section of the survey asked respondents to provide information about their screening practices. Participants were asked to indicate if they did or did not routinely screen for ASD and 61.3\% (19/31) of the EIs reported that they did not. Respondents who indicated that they did screen $(38.7 \% ; 12 / 31)$, were asked to signify what tool they typically used. All 12 of the participants who affirmed that they screened for ASD listed the M-CHAT-2 as the tool they used to conduct this procedure. Respondents who signified that they did not screen, were asked to indicate barriers to completing this process. Many of the professionals reported that they preferred to refer to a specialist. Others reported the following: not familiar with ASD screening tools, not adequately trained to conduct ASD-specific screenings, not confident recognizing the characteristics of ASD, lacked sufficient time, relied on clinical observations, and lacked adequate knowledge regarding resources and referral sources for ASD.

Given participants' responses to the ASK-Q, it was determined that their overall knowledge of ASD did improve after attending this training. Prior to the training, participants' correct responses averaged $87.7 \%$ across all survey statements. Following the workshop, participants' correct responses were 94.4\%. One of the largest changes in participant knowledge was on the statement "Autism occurs more commonly among higher socioeconomic and educational levels." Prior to the training, 23 disagreed with that statement; following the training, 31 disagreed. On the statement "Medication can alleviate the core symptoms of autism," 20 disagreed on the pre-training survey, following the training 28 disagreed. Given the statement "Autism can be diagnosed as early as 18 months," 23 participants agreed prior to training and 29 agreed post training. On the statement "Most children with autism may not look at things when you point at them," 20 agreed with this statement pre-training and 26 agreed post-training. When stated "It is important that all children diagnosed with autism receive some form of special education services at school," 20 people 
agreed with the statement prior to the training and 28 agreed at the conclusion of the workshop.

Part C EI providers demonstrated that they understood the value of early intervention for children with ASD. All 31 participants, on both the pre- and post-surveys, acknowledged that the earlier children received treatment for autism, the more effective the treatment; and, that early intervention could lead to significant gains in social and communication skills.

Providers were also well-informed on the general characteristics of autism. All 31 participants prior to and following training indicated that they recognized children with ASD may demonstrate the following behaviors: become excessively focused on one thing; show the need for routines and sameness; have strange reactions to the way things smell, taste, look, feel, or sound; and have trouble understanding others' facial expressions (see Table 2 for the results of the pre-test and post-test response totals for each of the 49 survey items).

Survey data was analyzed according to subdomains and it was determined the participants improved across the three knowledge subdomains. In the D/S subdomain, 30 responders were in the adequate knowledge range prior to the training and 31 demonstrated adequate knowledge post training. Participants also improved in the ET subdomain: 27 had adequate knowledge pre-training and 31 posttraining. For TR, 29 demonstrated adequate knowledge pre-training and 31 were within the adequate range following the training. In the ST subdomain, it was found that the participants did not endorse stigma related to ASD either prior to or following the training (see Table 3 for a summary of results related to each of the four subdomains).

\section{Discussion}

Based on the results of the ASK-Q survey responses for this study, EI providers' knowledge of ASD improved following completion of this training. EI providers had a high level of knowledge prior to the training and this level was enhanced after attending this workshop. Respondents' scores on the post-workshop ASK-Q improved in all three knowledge subdomains (i.e., diagnosis/symptoms, etiology, and treatment).

The data from this study revealed that this group of EI providers was well informed about the characteristics of ASD before attending the workshop. Yet, over half of the respondents reported that they were not comfortable conducting an ASD-specific screening, nor did they feel they had been adequately trained to do so. This result corresponded with other studies that sought to determine ASD screening barriers among pediatric providers [4], [7]. For these participants, it seemed that regardless of the strong knowledge base they had relative to the characteristics of ASD, they were not comfortable conducting an
ASD-specific screening without targeted training. This is an important finding for Part $\mathrm{C}$ administrators and coordinators to consider. It may be essential for EI providers to receive specific training to administer ASD screening tools. If this type of instruction is not provided, EIs may continue to rely on developmental screenings and/or non-specific clinical observations [11].

As discussed in the literature and observed in this project, it is imperative that EIs be given strategies for discussing the results of ASD screenings with caregivers [4], [13], [14], [15]. Professionals will benefit from guided practice on how to appropriately share screening outcomes with families, irrespective of the screening results. Further, Part $\mathrm{C}$ programs should identify appropriate community resources and referral sources that EI providers can discuss with families when the screening has been completed [4], [13], [14], [15]. By providing EIs with this information, they are better able to assist families to move forward with the ASD diagnostic process. Professionals' lack of knowledge regarding service and support options may also serve as a barrier to conducting screenings [14], [15]. Part C administration should take care to equip their providers with this type of valuable information.

Helping EI providers recognize the important role they play in the early identification of ASD is critical. Part $\mathrm{C}$ providers can become a front-line resource in the efforts to identify and diagnosis ASD as early as possible in a child's life [4]. For EI providers to respond to this important call, they must be equipped with the necessary knowledge and skills. While this study has a limited number of participants, these EI providers demonstrated that with targeted ASD training, they were able to achieve an adequate level of knowledge to effectively conduct ASD-specific screenings with children and families in Part $\mathrm{C}$ programs.

\section{Conclusion}

Evidence confirms that autism screening programs for young children continue to lag and children are being diagnosed at an age far beyond when their symptoms become evident [4]. It is vital, therefore, that we recognize and prepare professionals who can administer early and accurate screenings. The current study serves to support the literature that recommends communities consider providing ASD- specific trainings for EI providers [4]. In so doing, this may assist to break down the barriers that have reportedly detoured Part $\mathrm{C}$ providers from consistently engaging in early and systematic ASD-screening programs that correspond with the AAP guidelines. Productively activating this group of professionals may help to ensure children and families who are faced with negotiating the challenges of ASD will gain timely access to vital services and resources. 
Table 1. Demographic statistics for survey participants

\begin{tabular}{|c|c|c|}
\hline Variable & $N$ & Percentage \\
\hline \multicolumn{3}{|l|}{ Gender } \\
\hline Female & 29 & 93.5 \\
\hline Male & 2 & 6.5 \\
\hline \multicolumn{3}{|l|}{ Age } \\
\hline 25 to 34 & 10 & 32.3 \\
\hline 35 to 44 & 8 & 25.8 \\
\hline 45 to 54 & 6 & 19.4 \\
\hline 55 to 64 & 7 & 22.6 \\
\hline \multicolumn{3}{|l|}{ Discipline $^{\mathrm{a}, \mathrm{b}}$} \\
\hline Social worker & 3 & 10 \\
\hline Physical therapist & 5 & 16.7 \\
\hline Coordinator & 0 & 0.0 \\
\hline Occupational therapist & 5 & 16.7 \\
\hline Early Childhood Education/Unified/Special Education & 5 & 16.7 \\
\hline Speech-language pathologist & 9 & 30.0 \\
\hline Audiologist & 1 & 3.3 \\
\hline Not specified & 2 & 6.7 \\
\hline \multicolumn{3}{|l|}{ Years in practice } \\
\hline Less than or equal to 5 & 6 & 19.4 \\
\hline 6 to 10 & 6 & 19.4 \\
\hline 11 to 15 & 2 & 6.5 \\
\hline
\end{tabular}


16 to 20

21 to 25

Greater than or equal to 26

Routinely screen for ASD?

Yes

For those who screen $(n=12)$, what are the follow-up steps?d, e

Refer to specialist for follow-up

Monitor Symptoms and follow-up

Do comprehensive evaluation

Other: Depends on parent's wishes

For those who do not screen $(n=19)$, what are the reasons? ${ }^{\mathrm{d}, \mathrm{e}}$

Refer to specialists

Not familiar with screening tools

Lack of sufficient training

Do not feel confident to identify characteristics related to ASD

Lack of sufficient time

Rely only on clinical observations

Lack of adequate knowledge about resources and referral sources for ASD

${ }^{a}$ All 31 participants identified their practice setting as homebased.

${ }^{\mathrm{b}}$ Only 30 participants responded to this question.

${ }^{\mathrm{c}}$ All 12 participants who screen reported using the MCHAT as the screening tool.

${ }^{\mathrm{d}}$ Participants could select more than one option.

${ }^{\text {e }}$ Percentiles reflect subgroups ( $n=12$ for screeners; $n=19$ for non-screeners). 
Table 2. Results for pre-test and post-test correct response totals for each item per subdomain

$\begin{array}{cccc}\text { Item } & \begin{array}{c}\text { Pre-test correct } \\ (\%)\end{array} & \begin{array}{c}\text { Post-test } \\ \text { correct (\%) }\end{array} & \text { Change }\end{array}$

\section{Diagnosis/symptoms}

28. Most children with autism may not look at things when you point at them.

\begin{tabular}{|c|c|c|}
\hline $20(64.5)$ & $26(83.9)$ & 6 \\
\hline $23(74.2)$ & $29(93.5)$ & 6 \\
\hline 25 (80.6) & $29(93.5)$ & 4 \\
\hline $26(83.9)$ & $29(93.5)$ & 3 \\
\hline $27(87.1)$ & $30(96.8)$ & 3 \\
\hline $28(90.3)$ & $30(96.8)$ & 2 \\
\hline 29 (93.5) & $31(100.0)$ & 2 \\
\hline $26(83.9)$ & $27(87.1)$ & 1 \\
\hline $30(96.8)$ & $31(100.0)$ & 1 \\
\hline $31(100.0)$ & $31(100.0)$ & 0 \\
\hline $31(100.0)$ & $31(100.0)$ & 0 \\
\hline $30(96.8)$ & $30(96.8)$ & 0 \\
\hline $31(100.0)$ & $31(100.0)$ & 0 \\
\hline $30(96.8)$ & $30(96.8)$ & 0 \\
\hline $21(67.7)$ & $21(67.7)$ & 0 \\
\hline $31(100.0)$ & $31(100.0)$ & 0 \\
\hline $30(96.8)$ & $29(93.5)$ & -1 \\
\hline
\end{tabular}

3. Some children with autism may lose acquired speech.

sameness.
their arms.
gressive or hyperactive.
Etiology one thing.

36. Autism occurs more commonly among higher socioeconomic and educational levels.

$\begin{array}{lll}23(74.2) & 31(100.0) & 8 \\ 4(12.9) & 10(32.3) & 6 \\ 25(80.6) & 31(100.0) & 6 \\ 27(87.1) & 31(100.0) & 4 \\ 27(87.1) & 30(96.8) & 3 \\ 27(87.1) & 30(96.8) & 3 \\ 28(90.3) & 31(100.0) & 3 \\ 28(90.3) & 30(96.8) & 2\end{array}$

26. Vaccinations cause autism.

44. Many children with autism have difficulty using everyday language to communicate their needs.

10. Some children with autism do not talk.

54. Many children with autism get upset if their routine is changed.

18. All children with autism usually have problems with aggression.

29. Some children with autism show intense interest in parts of objects.

6. Children with autism may have strange reactions to the way things smell, taste, look, feel,

7. Many children with autism have trouble understanding facial expressions

15. Many children with autism have trouble tolerating loud noises or certain types of touch

25. Many children with autism show the need for routines and sameness.

34. Many children with autism repeatedly spin objects or flap their arms.

41. A lot of children with autism have problems with being aggressive or hyperactive.

35. Autism is a communication disorder.

53. The cause of autism is not yet known for sure.

16. Autism is more frequently diagnosed in males than females.

13. Autism happens mostly in middle class families.

14. Autism is preventable. ${ }^{\mathrm{a}}$

51. Autism is something that is caused by God or a supreme being. ${ }^{\text {a }}$ 
47. Traumatic experiences very early in life can cause autism.

$\begin{array}{lll}25(80.6) & 27(87.1) & 2 \\ 29(93.5) & 31(100.0) & 2 \\ 29(93.5) & 31(100.0) & 2 \\ 30(96.8) & 31(100.0) & 1 \\ 31(100.0) & 31(100.0) & 0 \\ 31(100.0) & 31(100.0) & 0 \\ 27(87.1) & 26(83.9) & -1\end{array}$

\section{Treatment}

9. It is important that all children diagnosed with autism receive some form of special education services at school. ${ }^{\mathrm{a}}$

$20(64.5) \quad 28(90.3) \quad 8$

11. Medication can alleviate the core symptoms of autism.

$20(64.5) \quad 28(90.3) \quad 8$

12. There is currently no cure for autism.

$31(100.0)$

8. We now have treatments that can cure autism.

$26(83.9)$

$29(93.5) \quad 3$

38. Behavior therapy is an intervention most likely to be effective for children with autism.

49. With the proper treatment, most children diagnosed with autism eventually outgrow the disorder.

42. Children with autism cannot learn any social skill.

$30(96.8) \quad 31(100.0) \quad 1$

22. The earlier treatment of autism starts, the more effective it tends to be.

$31(100.0) \quad 31(100.0) \quad 0$

27. Most children with autism are extremely impaired and cannot live independently as adults. $^{\mathrm{a}}$

$29(93.5) \quad 29(93.5) \quad 0$

39. Early intervention can lead to significant gains in social and communication skills of children with autism.

17. Children with autism can grow up to live independently.

$31(100.0) \quad 30(96.8) \quad-1$

45. Early intervention demonstrates no additional benefit to children with autism.

\section{Stigma}

5. There is a negative opinion towards children diagnosed with autism in some communities.

$\begin{array}{lll}27(87.1) & 31(100.0) & 4 \\ 30(96.8) & 31(100.0) & 1 \\ 30(96.8) & 31(100.0) & 1 \\ 30(96.8) & 31(100.0) & 1\end{array}$
showing symptoms of autism.

${ }^{\text {a }}$ These seven items $(9,14,18,27,32,51,55)$ were used to calculate the Stigma subdomain score. 
Table 3. Knowledge and stigma endorsement results for survey participants by subdomain

\begin{tabular}{|c|c|c|}
\hline ASK-Q subtest ${ }^{\mathrm{a}}$ & Pre-test $(\%)$ & Post-test (\%) \\
\hline \multicolumn{3}{|c|}{ Diagnosis/symptoms } \\
\hline Inadequate knowledge $(0-10)$ & $1(3.2)$ & $0(0.0)$ \\
\hline Adequate knowledge (11-17) & $30(96.8)$ & $31(100.0)$ \\
\hline \multicolumn{3}{|c|}{ Etiology } \\
\hline Inadequate knowledge $(0-10)$ & $4(12.9)$ & $0(0.0)$ \\
\hline Adequate knowledge (11-15) & $27(87.1)$ & $31(100.0)$ \\
\hline \multicolumn{3}{|c|}{ Treatment } \\
\hline Inadequate knowledge (0-9) & $2(6.5)$ & $0(0.0)$ \\
\hline Adequate knowledge (10-13) & $29(93.5)$ & $31(100.0)$ \\
\hline \multicolumn{3}{|c|}{ Stigma $^{\mathrm{b}}$} \\
\hline Endorse stigma (0-2) & $0(0.0)$ & $0(0.0)$ \\
\hline Do not endorse stigma (3-7) & $31(100.0)$ & $31(100.0)$ \\
\hline
\end{tabular}

${ }^{a}$ Numbers in parentheses in this column represent subdomain score ranges.

${ }^{\mathrm{b}}$ For the stigma endorsement sub score, a correct item score is equivalent to not endorsing the stigma.

\section{References}

[1] Center for Disease Control and Prevention. (2020, April 15). Data and statistics on autism spectrum disorder. https://www.cdc.gov/ncbddd/autism/data.html (Access Date: 24, August, 2020).

[2] Zwaigenbaum, L., Bauman, M., Stone, W., Nurit, Y., Estes, A., Hansen, R., McPartland, J., Natowicz., M., Choueiri, R., Fein, D., Kasari, C., Pierce, K., Buie, T., Carter, A., Davis, P., Granpeesheh, D., Mailloux, Z., Newschaffer, C., Robins, D., Roley, S., Wagner, S., and Wetherby, A., "Early identification of autism spectrum disorder: Recommendations for practice and research", Pediatrics, 136(1), 2015, pp. 810-840.

[3] Ben-Sasson, A., Atun-Einy, O., Yahav-Jonas, G., LevOn, S., and Gev., T., "Training physical therapists in early ASD screening", Journal of Autism and Developmental Disorders, 48, 2018, pp. 3926-3938.

[4] Stone, W., Ibanez, L., Carpentier, P., Posner, E., Bravo, A., Frederick, L., and Locke, J., "Early intervention providers' perspectives about working with families of toddlers with suspected ASD: A qualitative study", Journal of Autism and Developmental Disorders", 2019, Advance online publication. doi.org/10.1007/s10803-019-04337-x.

[5] Johnson, C., Myers, S., and American Academy of Pediatrics Council on Children with Disabilities, "Identification and evaluation of children with autism spectrum disorders", Pediatrics, 120(5), 2007, pp. 11833447.

[6] Hyman, S., Levy, S., Myers, S., and Council on Children with Disabilities, "Identification, evaluation, and management of children with autism spectrum disorder", Pediatrics, 145(1), 2020, pp. 1-7.

[7] Self, T., Parham, D., and Rajagopalan, J., "Autism spectrum disorder early screening practices: A survey of physicians", Communication Disorders Quarterly, 36(4), 2015, pp. 195-207.

[8] Crais, E., McComish, C., Humphreys, B., Watson, L., Baranek, G., Reznick, J., Christian, R., and Erls, M., "Pediatric healthcare professionals' view on autism spectrum disorder screening at 12-18 months". Journal of Autism and Developmental Disorders, 44, 2014, pp. 2311-2329.

[9] Baio, J., Wiggins, L., Christensen, D. et al., "Prevalence and characteristics of autism spectrum disorder among 
children aged 8 years - Autism and developmental disabilities monitoring network, 11 sites, United States, 014", Morbidity and Mortality Weekly Report Surveillance Summaries, 67(6), pp. 1-23.

[10] Self, T., Coufal, K., and Parham, D., "Allied healthcare providers' role in screening for autism spectrum disorders", Journal of Allied Health, 39(3), 2010, pp. 165-174.

[11] Tomlin, A., Koch, S., Ranches, C., Minshawi, N., and Swiezy, N., "Autism screening practices among early intervention providers in Indiana", Infants and Young Children, 26(1), 2013, pp. 74-88.

[12] Pizur-Barnekow, K., Muusz, M., McKenna, C., O'Connor, E., and Cutler, A., "Service coordinators' perceptions of autism-specific screening and referral practices in early intervention", Topics in Early Childhood Special Education, 33(3), 2013, pp. 153-161.

[13] Harrison, A., Bradshaw, L., Naqvi, N., Paff, M., and Campbell, J., "Development and psychometric evaluation of the autism stigma and knowledge questionnaire (ASK-Q)", Journal of Autism and Developmental Disorders, 47, 2017, pp. 3281-3295.

[14] Kobak, K., Stone, W., Ousley, O., and Swanson, A., "Web-based training in early autism screening: Results from a pilot study. Telemedicine and E-Health, 17(8), 2011, pp. 640-644.

[15] Kairys S., and Petrova, A., "Role of participation of pediatricians in the activated autism practice program in practicing children with autism spectrum disorders at the primary care setting", Global Pediatric Health, 3, 2016, pp. $1-6$.

[16] Robins, D., Casagrande, K., Barton, M., Chen, A., Dumont-Mathieu, T., and Fein, D., "Validation of the Modified Checklist for Autism in Toddlers, Revised with Follow-up (M-CHAT-R/F)", Pediatrics, 133(1), 2017, pp. $37-45$. 